\title{
Guidelines Program of the Brazilian Medical Association
}

1. Tenured Professor at the Faculty of Medicine of USP, Av. Dr. Arnaldo, 455 - Cerqueira César, São Paulo - SP, Brasil 2. Cordinator of the Guidelines Program of the Brazilian Medical Association, Rua São Carlos do Pinhal, 324 - Bela Vista, São Paulo - SP, Brasil

The Guidelines Program of the Brazilian Medical Association (AMB) has developed over 1,000 guidelines over the past 15 years, publishing only those that are maintained updated. Over the past 12 months, about 150 new themes were developed in a partnership with the Societies of Specialties of AMB.

This special supplement of the Journal of the Brazilian Medical Association (RAMB) aims at presenting a small sample of five different topics, content, and formats of guidelines recently developed.

As for the topics, they can help in decision making relating to diagnostic accuracy, therapeutic effectiveness, and clinical follow-up, listing the risk of adverse reactions.

The content always brings key elements, such as matters relevant to the topic, search strategies for the several databases used, eligibility criteria for the studies included, critical evaluation of the risk of bias, analysis of the strength of the evidence and of the grades for recommendation, closing with a summary of the evidence that supports the recommendations $\mathbf{s}^{1,2,3}$.

Concerning the format, recommendations may be accompanied and supported by results in the form of meta-analysis, with several strengths of evidence for each important outcome considered.

The development using an evidence-based methodology together with the Societies of Specialties allows for the due integration of scientific evidence and acquired clinical experience, with the primary goal of providing information to support equality and quality in healthcare, with an impact on the Brazilian health system.

The Guidelines Program strives to incorporate in its design methodology mechanisms to overcome the main barriers, balancing the different interests involved in healthcare in Brazil and constantly updating itself, whether regarding existing topics, or new ones.

The methodology for developing the guidelines strictly follows international standards (the program is affiliated to Guideline International Network - GIN) for developing evidence-based guidelines, which follows three basic premises ${ }^{1-3}$ :

1. They are based on the answers to specific clinical questions using systematic reviews in the scientific medical literature. In these reviews, the source and strength (level of uncertainty) of all scientific in- 
formation (evidence) used in the recommendations are transparent and explicit;

2. They take into account the experience accumulated by the coauthor doctors along with that of specialized professionals for criticism and translation of evidence;

3. They take into account the reality of the Brazilian population and the national health system, proposing new procedures or technologies with discernment and fairness.

In this special Supplement, we bring eleven topics: 1. Overactive bladder - pharmacological treatment; 2 . Moderate to severe plaque psoriasis - treatment with immunobiologics; 3. Relapsed ovarian cancer - diagnosis using 18F-FDG PET/CT; 4. Pelvic congestion syndrome - treatment with pelvic varicose veins embolization; and 5. Decompensated congestive heart failure - treatment with levosimendan; 6. Moderate to severe plaque psoriasis - treatment with drugs of the classic scheme; 7. Interstitial cystitis - intravesical treatment; 8. Deep brain stimulation in Parkinson Disease; 9. Ulcerative Rectocolitis - treatment with biologics; and 10. Crohn's Disease - treatment with biologic drugs and 11. Cervical cancer - staging and restaging with ${ }^{18} \mathrm{~F}$-FDG PET/CT

These topics have been disseminated in various segments of the healthcare system in an attempt to optimize their implementation process. We hope they are useful and can illustrate the methodological quality of guidelines based on scientific evidence.

\section{REFERENCES}

1. Bernardo WM. Cheers!!! Rev Assoc Med Bras (1992) 2016; 62: 809810. PMID: 28001249

2. Bernardo WM, Carneiro AV, Baracat EC. [Guidelines: unity and balance] Rev Assoc Med Bras (1992). 2009; 55: 498-9. PMID: 19918638.

3. Bernardo WM. [Clinical guidelines in Brazilian health system]. Rev Assoc Med Bras (1992) 2008; 54:377. PMID: 18989548. 